\title{
Therapeutic Modulation of Gut Microbiota in Functional Bowel Disorders
}

\begin{abstract}
Hyo Jeong Lee, Jong Kyoung Choi, ${ }^{2}$ Han Seung Ryu ${ }^{3}$ Chang Hwan Choi, ${ }^{4}$ Eun Hee Kang, ${ }^{1}$ Kyung Sik Park, ${ }^{5}$ Yang Won Min, ${ }^{6 *}$ and Kyoung Sup Hong ${ }^{7 *}$

${ }^{1}$ Health Screening and Promotion Center, Asan Medical Center, University of Ulsan College of Medicine, Seoul, Korea; ${ }^{2}$ Department of Internal Medicine, National Medical Center, Seoul, Korea; ${ }^{3}$ Department of Internal Medicine, Wonkwang University College of Medicine and Digestive Disease Research Institute, Iksan, Korea; ${ }^{4}$ Department of Internal Medicine, Chung-Ang University College of Medicine, Seoul, Korea; ${ }^{5}$ Department of Internal Medicine, Keimyung University School of Medicine, Daegu, Korea; ${ }^{6}$ Department of Medicine, Samsung Medical Center, Sungkyunkwan University School of Medicine, Seoul, Korea; and ${ }^{7}$ Department of Internal Medicine and Liver Research Institute, Seoul National University College of Medicine, Seoul, Korea
\end{abstract}

Functional bowel disorders (FBDs) are functional gastrointestinal disorders with symptoms attributable to the middle or lower gastrointestinal tract. These include irritable bowel syndrome, functional bloating, functional constipation, functional diarrhea, and unspecified FBD. Increasing evidence has emerged of late that intestinal microbiota is involved in the pathogenesis of FBDs. In this review, the therapeutic benefits and future perspectives of the currently available strategies for modifying the gut microbiota in FBDs are described, focusing primarily on irritable bowel syndrome and functional constipation.

(J Neurogastroenterol Motil 2017;23:9-19)

Key Words

Diet; Fecal microbiota transplantation; Irritable bowel syndrome; Microbiota; Probiotics

\section{Introduction}

Functional bowel disorders (FBDs) are functional gastrointestinal disorders with symptoms attributable to the middle or lower gastrointestinal tract. ${ }^{1}$ These include irritable bowel syndrome (IBS), functional bloating, functional constipation (FC), functional diarrhea, and unspecified FBD. ${ }^{1}$ The FBDs are among the most common gastrointestinal complaints, and they are associated with reduced quality of life. ${ }^{2,3}$ The pathophysiological mechanisms underlying these disorders are still incompletely understood, but they may be multifactorial, including visceral hypersensitivity, abnormal gastrointestinal motility, altered brain-gut axis, psychological disturbances, and low-grade inflammation. ${ }^{4,5}$ Increasing evidence has

Received: August 8, 2016 Revised: None Accepted: October 30, 2016

(c) This is an Open Access article distributed under the terms of the Creative Commons Attribution Non-Commercial License (http://creativecommons. org/licenses/by-nc/4.0) which permits unrestricted non-commercial use, distribution, and reproduction in any medium, provided the original work is properly cited.

*Correspondence: Yang Won Min and Kyoung Sub Hong are equally responsible for this work. Yang Won Min, MD, PhD

Department of Medicine, Samsung Medical Center, Sungkyunkwan University School of Medicine, 81 Irwon-ro, Gangnam-gu, Seoul 06351, Korea

Tel: +82-2-3410-3409, Fax: +82-2-3410-6983, E-mail: yangwonee@gmail.com

Kyoung Sup Hong, MD, PhD

Department of Internal Medicine, Seoul National University Hospital, Seoul National University College of Medicine, 101 Daehak-

ro, Jongno-gu, Seoul 03080, Korea

Tel: +82-2-2072-0360, Fax: +82-2-762-9662, E-mail: kshong1@snu.ac.kr

Hyo Jeong Lee and Jong Kyoung Choi contributed equally to this work. 
emerged of late that intestinal microbiota is involved in the pathogenesis of FBDs. ${ }^{6}$

The phenomenon of post-infectious IBS, small-intestinal bacterial overgrowth implicated in the subgroups of IBS, and alteration of the fecal microbiota of IBS patients directly support the importance of gut microbiota in IBS pathophysiology. ${ }^{6-12}$ The known physiologic effects of altered microbiota include bile salt deconjugation changes leading to changes in stool volume and consistency, as well as bacterial fermentation changes resulting in alterations in the gas volume and/or composition. These alterations indirectly support the role of gut microbiota in the development of FBDs. ${ }^{8}$

The treatment of FBDs remains challenging. An improved understanding of host-microbiota interactions in FBDs is important not only for its pathogenesis but also for enabling therapeutic modulation of the microbiota. ${ }^{13}$ The potential therapeutic approaches, including dietary manipulation, prebiotics, probiotics, synbiotics, antibiotics, and fecal microbiota transplantation, are attempts to modify the gut microbiota. ${ }^{6}$ In this review, the therapeutic benefits and future perspectives of the currently available strategies for modifying gut microbiota in FBDs are described, focusing primarily on IBS and FC.

\section{Diet and Intestinal Microbiota}

As the knowledge about the human gut microbiome has advanced, the composition of the microbiota has been discovered to have a profound impact on human health. ${ }^{14,15}$ The human gut microbiota is influenced by various factors. Of these, diet is a key element determining its composition. ${ }^{15,16}$ Carbohydrate fermentation is a core activity of the human gut microbiota, driving the energy and carbon economy of the colon. ${ }^{17,18}$ Short-chain fatty acids (SCFAs), which are the main end products of bacterial metabolism, regulate the intestinal physiology and immune function, and act as a substrate for lipogenesis and gluconeogenesis. ${ }^{17}$ Therefore, it is recognized that certain foods may affect the microbiota activities linked to the systemic host physiology.

For IBS patients, food is an important issue in the management of their symptoms. A large number of IBS patients consider their symptoms related to food, and two-thirds of IBS patients report restricting their diets. ${ }^{19-23}$ Many different dietary approaches for the management of the IBS symptoms have been tried over the years. $^{24}$ There are many mechanisms by which food may act on the gut to cause symptoms of IBS. ${ }^{23,25}$ Among these, altered microbial composition and functions of food may be important factors in the development of IBS symptoms. Thus, therapeutic dietary interven- tions that modify gut microbiota may be effective for alleviating IBS symptoms. In this section, the evidence for a link among diet, gut microbiota, and IBS symptoms are reviewed.

There is emerging strong evidence supporting diets for the IBS patients that are low in fermentable oligosaccharides, disaccharides, monosaccharides, and polyols (FODMAPs). Randomized controlled trials (RCTs) reported that a diet low in FODMAPS effectively reduced IBS symptoms, and the effects were comparable with those of the traditional IBS dietary advice. ${ }^{26,27}$ FODMAPs exacerbate IBS symptoms through various mechanisms, such as by increasing the small-intestinal water volume through an osmotic effect, colonic-gas production by bacterial fermentation, and altered intestinal motility. ${ }^{28}$ Carbohydrates fermented by the gut microbiota cause increased production of luminal gases, such as hydrogen, methane, and carbon dioxide. ${ }^{29}$ Excess hydrogen leads to luminal distension, resulting in increased bloating and borborygmi in those with visceral hypersensitivity. ${ }^{30}$ An unblinded study found that when challenged with a high-fermentable-carbohydrate "flatulogenic" diet, the frequency and volume of flatulence and other gastrointestinal symptoms increased compared with the baseline in both flatulence patients and the healthy controls. ${ }^{31}$ Moreover, flatulogenic diet revealed instability of the microbial composition and reduction of the microbial diversity in patients complaining of flatulence, whereas healthy controls' microbiota were stable. This study indirectly demonstrated the impact of the diet-microbiota interactions on the development of symptoms in IBS patients.

Methanogenic archaea, a major mechanism of hydrogen disposal in the human colon, may also contribute to the pathology of IBS. ${ }^{32}$ In previous studies, IBS patients had lower numbers of methanogenic bacteria compared with healthy controls, suggesting that IBS patients lack the functions for hydrogen removal. ${ }^{11,33}$ Methane also induces slow intestinal transit, and has been associated with constipation-dominant IBS (IBS-C). ${ }^{34}$ A study with 19 IBS-C patients found that Methanobrevibacter smithii is the predominant methanogen, and that the quantity of $M$. smithii in the stool correlated with the amount of methane excreted in the breath. ${ }^{35}$ The direct evidence for the role of methane in the diet-microbial interaction in IBS patients, however, is limited; thus, additional studies are warranted.

SCFAs are the main products of FODMAP fermentation, but the alteration or effect of SCFAs in IBS remains unknown. One case control study reported that IBS patients had higher levels of acetic acid and propionic acid than controls, and that the levels of acetic acid and propionic acid were associated with IBS symptom severity. ${ }^{36}$ It is not clear, however, if this alteration of the SCFAs was 
the simple result of the diet or the causal factor of IBS symptoms; therefore, further evaluation of the causal links among the FODMAPs, SCFAs, gut microbiota, and IBS symptoms is needed.

Although many clinical studies have demonstrated the effect of a low-FODMAP diet on symptoms of IBS, the impact of lowFODMAP intervention on the gut microbiota and its association with IBS symptoms have not been well evaluated. Two studies have investigated the impact of low-FODMAP diet on the gut microbiota in adult patients. ${ }^{37,38}$ The first randomized controlled study, with 41 IBS patients, found that more patients in the intervention group (low-FODMAP-diet group) reported adequate symptom alleviation compared with controls (habitual-diet group). Also, IBS patients had lower concentrations and proportions of Bifidobacterium than controls. ${ }^{37}$ A randomized crossover study with 27 IBS patients and 6 healthy subjects found that low-FODMAP diet reduced the total bacterial abundance, and the typical Australian diet increased the relative abundance of butyrate-producing Clostridium cluster XIVa and mucus-associated Akkermansia muciniphila bacterial groups that may have some health benefits. ${ }^{38}$ These studies showed the contradictory responses of gut microbiota and IBS symptoms to low-FODMAP diet. Further studies are warranted to elucidate the role of altered microbiota through the restriction of FODMAPs in the alleviation of IBS symptoms and its long-term effect on the general health of IBS patients.

Dietary fat may play a role in the pathogenesis of IBS. ${ }^{39} \mathrm{~A}$ substantial number of people with IBS report that their symptoms are associated with fatty food, and that they frequently avoid fatty foods to alleviate the symptoms. ${ }^{19,21,39,40}$ In people with IBS, the lipid-induced inhibition of gut-propulsive motility is up-regulated, and the colonic hypersensitivity in response to the duodenal lipid infusion is exacerbated. ${ }^{39,41,42}$ These effects may be associated with various IBS symptoms. The altered composition of the intestinal microbiota by dietary fat may also be responsible for IBS symptoms. In IBS patients, there is a strong mucosal immune activation predominantly characterized by activated $\mathrm{T}$ lymphocytes and mast cells, along with evidence of an increased release of mediators known to signal to epithelial, neuronal, and muscle cells, leading to intestinal dysfunction. The immune activation in low-grade inflammation may be the consequence of the perturbation of gut microbiota. ${ }^{43} \mathrm{~A}$ highfat diet alters the composition of the intestinal microbiota, and this promotes intestinal inflammation. ${ }^{44-47}$ In one study, a high-fat diet induced increasing ileal tumor necrosis factor alpha mRNA only in conventionally raised, specific-pathogen-free mice and not in germfree mice. ${ }^{46}$ It changes the behavior as well as the composition of the intestinal microbiota in genetically susceptible hosts, and promotes inflammation. ${ }^{47}$

Dietary fat also increases the excretion of bile acids. The intestinal microbiota involved in bile acid metabolism, and increased colonic bile acid exposure, may induce IBS symptoms by stimulating colonic motility and secretion, and modulating the microbial composition. ${ }^{48,49} \mathrm{~A}$ significant increase in fecal primary bile acid and dysbiosis in the feces involved in bile acid transformation were noted in people with diarrhea-dominant IBS (IBS-D). ${ }^{48} \mathrm{~A}$ diet high in saturated-milk-derived fatty acids increased the formation of taurine-conjugated bile acids in genetically susceptible $\mathrm{Il10}^{-/-}$mice, promoting the expansion of a low-abundance, sulphite-reducing pathobiont, Bilophila wadsworthia, leading to colitis. ${ }^{50}$ There have been no well designed studies, however, that have investigated the effect of the altered gut microbiota on IBS symptoms by restricting dietary-fat intake.

Dietary proteins are also related to IBS symptoms. An example is gluten, which is regarded as a major issue in IBS. Recent studies found that a large number of IBS patients suffer from non-celiac gluten sensitivity. ${ }^{51}$ Gluten causes IBS symptoms in people with IBS, and a gluten-free diet reduces IBS symptoms in people with IBS-D. ${ }^{52,53}$ Gluten generates IBS symptoms thorough altered gut permeability, tight-junctional biology, and enhanced systemic immune responses. ${ }^{53}$ There is no evidence, however, of the impact of gluten intake on intestinal microbiota, and of its association with IBS symptoms.

Targeting the diet and microbiota remains an attractive option in treating or preventing FBDs. In the future, engineered diets that restrict deleterious components but supplement beneficial nutrients can be used to modify the luminal intestinal environment for the treatment or prevention of diseases. ${ }^{54}$ Human dietary-intervention studies in FBDs are scarce, however, because a dietary-intervention study has many limitations, such as the inability to use placebos as controls and the difficulty of accurately capturing the dietary intake. Many clinical studies on diets have been limited to specific nutrients and foods, but the interactions between the foods consumed, the proportion of food intake, and the effect of the food structure and/ or cooking practices should also be considered. ${ }^{16}$

The development of therapeutic dietary strategies for modifying the gut microbiota for FBDs is still in the beginning stage. More fundamental studies on the effect of diet on gut microbiome and the pathogenesis of FBDs are needed. A common agreement is also needed on what constitutes a healthy population of gut microbes, and inter-individual differences in the populations of gut microbiota should be considered. ${ }^{15,16}$ Further studies to elucidate the interplay of diet, microbiota, genetic factors, the host immune system, and the 
symptoms of FBD are also warranted. The compositional change of the gut microbiota, as well as the gene expression profiles and functionality, should be proven. An investigation of the role of gut microbiomes as biomarkers for dietary intake, disease development, and/or treatment response would be interesting.

\section{Pre- and Probiotics in Functional Bowel Disorders}

Lactobacillus and Bifidobacterium are the representative components of commercialized probiotic products. There are plenty of literatures that assess the efficacy and safety of these 2 probiotic genera. On the other hand, there are still few literatures that assess other genuses, such as probiotics Escherichia or Saccharomyces. Combination products containing 2 or more probiotic species have become the mainstream of late. "Strain specificity" means that a species of probiotics can have a different level of anti-inflammatory property or resistance to gastric acid or bile salt. ${ }^{55}$ It is reasonable that a probiotic species can show a wide spectrum of efficacy levels in IBS patients according to the source that determines the strain as a subtype of the species. The proven efficacy of one strain of a species cannot be extrapolated to other strains of the species. In the same context, the "non-efficacy of a strain of a species" does not mean that the other strains of the species cannot be effective. A probiotic strain that has been proven to be effective through RCTs may not be available in a certain country at a specific time. Moreover, probiotic products are classified not as drugs but as food items in most countries. In this case, the efficacy data is not obligatory for marketing approval. Clinicians should be aware of the scientific evidence before using probiotic products. There may be no clinical data, however, about a specific strain or a combination product. A clinical decision based on the comprehensive knowledge about the efficacy of probiotics can be essential.

\section{Global Efficacy of Probiotics in Irritable Bowel Syndrome: Dichotomous Assessment by Patients}

A dichotomous question about the global efficacy or persistence of symptoms may be difficult to answer for patients, and may be substantially influenced by recall bias, but it seems to be a good indicator of patient satisfaction. Ford et $\mathrm{al}^{56}$ performed a comprehensive meta-analysis of 23 placebo-controlled RCTs with IBS patients and reported that the relative risk of IBS symptoms persisting or remaining unalleviated after treatment with probiotics vs placebo was 0.79 (95\% CI, 0.70-0.89). Although a significant effect was observed, a statistically significant heterogeneity was detected among the studies, which may be partially due to the different proportions of IBS subtypes. The consensus agreement and the grade of the evidence for the effect of the probiotics were lower in IBS-C than in IBS-D. ${ }^{57}$

\section{Abdominal Pain and Discomfort: Global Irritable Bowel Syndrome or Abdominal-pain Scores}

A meta-analysis of 24 RCTs presenting the global IBS or abdominal-pain scores reported a significant symptom alleviation through a pooled analysis with 2001 patients. ${ }^{56}$ No significant effect was observed, however, in the per-strain or per-product analyses of 2 to 3 RCTs, respectively: L. plantarum DSM $9843^{58,59}$; B. infantis $35624^{60,61}$; VSL \# $3^{62-64}$; a combination product of $L$. paracasei subspecies paracasei $\mathrm{F} 19$, L. acidophilus La5, and B. lactis $\mathrm{Bb} 12^{65-67}$; and the other combination of B. lactis DN-173 010, S. thermophilus, and L. bulgaricus. ${ }^{68,69}$

A systematic review of 18 RCTs involving 1806 IBS patients and consensus voting reported a high grade of evidence for the effect of probiotics on abdominal pain, and a good consensus agreement. ${ }^{57}$ The abdominal pain was significantly alleviated in 5 of the 6 RCTs in which abdominal pain was assessed as a primary outcome variable, but in only 3 of the 12 RCTs in which abdominal pain was evaluated as a secondary variable. ${ }^{57}$ Unexpectedly, the Lactobacillus plantarum MF 1298 strain, which had been known to increase the expression of the tight-junction protein through an in vitro study, was reported to aggravate the symptoms, including abdominal pain, discomfort, urgency, and diarrhea, after 3-week treatment in 16 IBS patients. $^{70}$

\section{Bloating and Flatulence}

A meta-analysis of 17 separate RCTs involving 1446 patients showed a significant effect of probiotics on the bloating scores. ${ }^{56}$ An international guide through a systematic review and consensus voting reported $70 \%$ agreement and a moderate grade of evidence for the effect of certain probiotics on bloating in people with IBS. ${ }^{57}$

There were $10 \mathrm{RCTs}$ involving 741 patients who reported a significant effect of probiotics on their flatulence scores. An RCT reported the effect of Lactobacillus reuteri on the passing of gases in 54 patients after 6-month administration. There was no significant effect on the abdominal-pain or sum scores. The flatulence scores 
decreased consistently, however, for 6 months in the treatment group compared to the placebo. ${ }^{71}$ Although the above-mentioned study showed negative results, it may be an example of a study that showed a promising probiotic strain that could effectively alleviate a specific symptom of IBS.

\section{Urgency, Bowel Habit, Borborygmi, and Feeling of Incomplete Evacuation}

Six RCTs reported no significant benefit of probiotics on the urgency scores. ${ }^{56}$ There is a still controversy about the effect of probiotics on the bowel habits, including the stool consistency and the frequency of defecation. Of the $2 \mathrm{RCT}$ in which the bowel habits were assessed as the primary outcome variable, Kim et al ${ }^{62}$ evaluated the IBS symptoms and gastrointestinal transit times in 25 IBS$\mathrm{D}$ patients before and after the treatment with VSL\#3 for 8 weeks. Although the bloating was significantly alleviated, there was no additional effect over placebo on the frequency of bowel movement, stool consistency, gastric emptying time, and colonic transit time. In a study about 6-month treatment with a probiotic mixture containing Lactobacillus rhamnosus GG, L. rhamnosus LC705, Bifidobacterium breve Bb99, and Propionibacterium freudenreichii spp. shermanii JS in 103 IBS patients, there were significant effects on the global abdominal symptoms, borborygmi, bowel urgency, and feeling of incomplete evacuation, but there was no change in the bowel habits. $^{72}$ Six of the 14 RCTs in which the bowel habits were assessed as a secondary outcome variable revealed no changes in the bowel frequency and stool consistency. ${ }^{57}$ The above-mentioned RCT with the L. plantarum MF 1298 strain showed a significant deterioration of diarrhea and urgency in IBS-D. ${ }^{70}$

\section{Colonic Transit Time and Constipation}

There were several RCTs that evaluated the effect of probiotics on the colonic transit time in constipation. In 36 healthy females, the whole and sigmoid colonic transit time significantly decreased after the ingestion of a fermented milk product containing the Bifidobacterium lactis DN-173 101 strain 3 times a day for 10 days. ${ }^{73}$ There was a significant decrease in the whole and right-side colonic transit time as well as an improvement in the abdominal-pain and bowel urgency scores after the ingestion of a fermented milk product containing the $B$. lactis DN-173010 strain twice a day for 4 weeks in 34 IBS-C patients, but there was no change in the defecation frequency, stool consistency, and defecation discomfort such

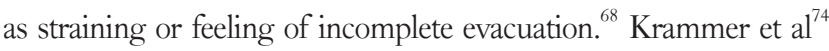

reported that the colonic transit time and the subjective symptoms of constipation improved after the treatment with a yoghurt containing Lactobacillus casei Shirota once a day for 4 weeks in 24 people with slow-transit constipation. The ingestion of a yoghurt combined with capsules containing $B$. lactis $\mathrm{HN} 019$ decreased the whole gut transit time in a dose-dependent manner, and reduced the frequency of the functional gastrointestinal symptoms, in 88 healthy volunteers with gastrointestinal symptoms. ${ }^{75}$ There are several limitations, however: the 3 RCTs evaluated only the short-term effect of fermented milk products and could not demonstrate the effect on the bowel habits, and the Rome III criteria for FC were not applied before the subject selection.

Four RCTs evaluating probiotics in 160 patients who had met the Rome criteria for FC were identified. ${ }^{76-79}$ An RCT on a fermented milk product containing $L$. casei Shirota did not show any improvement in the bowel habits or alleviation of the subjective symptoms of constipation. ${ }^{77}$ Two of the 4 RCTs showed an increase in the number of bowel movements after the treatment with probiotics, but there was no effect on the stool consistency, straining, and feeling of incomplete evacuation. ${ }^{76,78}$ Riezzo et $\mathrm{al}^{79}$ reported that a probiotic-enriched artichoke containing Lactobacillus paracasei IMPC 2.1 significantly improved the hard-stool, bowel movement frequency, and feeling-of-incomplete-evacuation scores compared with the control artichoke. Artichoke is a vegetable that is rich in inulin-type fructan, which is one of the representative prebiotics. Apart from improvements in the bowel habits and alleviation of the subjective symptoms of constipation, this study on synbiotics also revealed a significant increase in the concentration of fecal propionic acid, which is a kind of SCFA. ${ }^{79}$ These results warrant future studies about the ideal symbiotic combinations as both safe and sustainable treatment options for constipation.

\section{Safety of Probiotics in Gastrointestinal Disorders}

Although probiotics have an excellent overall safety record, they should be used with caution in certain patient groups, particularly in neonates born prematurely or with immune deficiency. ${ }^{80}$ Fears that live probiotic organisms may lead to systemic sepsis have been allayed by the absence of such reports from studies among people with inflammatory bowel disease (IBD) where the intestinal barrier may be compromised. Two notes of caution must be mentioned herein. ${ }^{81}$ The first relates to reports of septicemia occurring among infants with short bowel syndrome, ${ }^{82}$ and the second concerns instances of increased mortality among people with severe acute 
pancreatitis who had been administered a probiotic cocktail through a nasogastric tube. These deaths were associated not with sepsis but with intestinal ischemia, whose etiology remains unclear. ${ }^{83}$

\section{Fermented Milk: Probiotics vs Synbiotics vs High-FODMAP Foods}

Prebiotics are defined as non-digestible but fermentable foods that beneficially affect the host by selectively stimulating the growth and activity of one species or a limited number of species of bacteria in the colon. The oligosaccharide in human breast milk is considered the prototypic prebiotic as it facilitates the preferential growth of Bifidobacterium and lactobacilli in the colons of breastfed neonates. ${ }^{81}$ To some degree, a fermented milk product is required to contain lactose, which is a poorly digestible disaccharide in most adults. Moreover, fructo-oligosaccharide is often added to the bacterial yoghurts in the market, as a functional ingredient. It seems reasonable to regard commercialized bacterial yoghurts as synbiotics rather than probiotics. Since recently, a diet low in FODMAPs has often been used to manage the functional gastrointestinal symptoms in IBS patients. ${ }^{84}$ Fermented milk products, which are high in lactose or oligosaccharides, may be a major source of FODMAPs and are likely to aggravate the functional gastrointestinal symptoms in some people with IBS.

\section{Antibiotics in Functional Bowel Disorders -}

There have been various attempts to treat intestinal symptoms by using various antibiotics. ${ }^{85-87}$ Finally, in 2015, rifaximin became the first Food and Drug Administration-approved antibiotic for the treatment of IBS-D. Rifaximin is a rifamycin-based oral nonabsorbable antibiotic that has a strong in vitro antimicrobial effect against aerobic and anaerobic gram-positive and gram-negative bacteria. ${ }^{88}$ Rifaximin has high solubility in the presence of bile acids, and poorly systemic absorption. ${ }^{85,89,90}$ It may result in inhibition of a broad spectrum of bacteria in the small intestine, without a systemic adverse effect. ${ }^{91}$

Many previous studies reported that rifaximin treatment in SIBO and IBS patients had significant benefits compared with placebo. ${ }^{87,92-94}$ In the 2 largest phase 3 trials involving people with non-constipated IBS (TARGET 1 and 2), treatment with rifaximin $550 \mathrm{mg} 3$ times a day for 2 weeks achieved adequate alleviation of the global IBS symptoms ( $40.7 \%$ vs $31.7 \%$ for placebo, pooled; $P<0.001)$ and bloating (40.2\% vs $30.3 \%$ for placebo, pooled; $P$ $<0.001)$ during the first 4 weeks after treatment. ${ }^{87}$ In addition, a meta-analysis of 5 RCTs of rifaximin for IBS, including TARGET 1 and 2, reported that rifaximin was more efficacious than placebo for global IBS symptom alleviation (OR, 1.57; 95\% CI, 1.22) and bloating alleviation (OR, 1.55; 95\% CI, 1.23-1.96). ${ }^{94}$

Although it is generally acknowledged that rifaximin leads to short-term clinical improvements in some IBS patients, there are several points to be considered before promoting the widespread use of rifaximin. First, a less-than-half response rate and a $9 \%$ therapeutic gain means that several administrations are needed to treat roughly 11 patients. ${ }^{95}$ To prevent antibiotic misuse, a specific predictor of the response to antibiotic treatment in IBS patients is needed. The potential candidates for the predictor are diagnostic tests for SIBO, including breath tests and analysis of the uppergut aspirates. In previous studies, normalization of the results of the breath test in IBS patients after antibiotic therapy has been shown to be correlated with a successful response to antibiotic therapy. ${ }^{86,92,93,96}$ The predictive value of a positive lactulose breath test has been disappointing, however, in selecting patients who will respond. ${ }^{97-99}$ In a recent prospective study that used upper-gut aspiration culture for diagnosing SIBO, IBS patients with SIBO had greater symptom alleviation than patients without $\operatorname{SIBO}(87.5 \%$ vs $25 \%) 1$ month after the administration of antibiotics. ${ }^{100}$ The technique of upper-gut aspiration, however, has limited application in clinical practice due to its invasiveness and expensiveness. Further study will be required to identify the biomarker-based strategies that will enrich the likelihood of a response to antibiotics in IBS patients. ${ }^{95}$

Although a recent study suggested that long-term rifaximin use for hepatic encephalopathy is safe and tolerable, ${ }^{101}$ it must be considered that excessive rifaximin use may lead to resistance and adaptation among pathogens. There is emerging evidence that rifaximin use can select rifaximin-resistant strains of bacteria, such as Clostridium difficile and Escherichia coli. ${ }^{102,103}$ Furthermore, rifaximin is a structural analog of rifampin, which is used for treating several infections, such as tuberculosis and methicillin-resistant Staphylococcus aureus. ${ }^{104}$ In a prospective study, rifampin-resistant human-skin staphylococci emerged after oral intake of rifaximin by 20 healthy volunteers, and these isolates were still found on the hands and lower arms after 9 weeks. ${ }^{104}$ There is a consensus that rifaximin may be a treatment of choice for some IBS patients. Before the promotion of the wide use of rifaximin, however, further studies are still needed for the identification of the subpopulation of IBS patients who may best benefit from rifaximin use. 


\section{Fecal Microbiota Transplantation}

In people with disease related to dysbiosis, reconstituting the normal gut microbiota is essential to the treatment of the disease. The administration of a fecal suspension containing the normal gut microbiota from a healthy donor to the gut of the dysbiosis patient, known as fecal microbiota transplantation (FMT), can restore the phylogenetic diversity and microbiota more typical of a healthy person. As the disrupted gut microbiota is a key factor in the pathogenesis of $C$. difficile infection (CDI), FMT is increasingly used for recurrent or severe CDI. ${ }^{105-107}$ In a recent systemic review involving 516 patients with recurrent $\mathrm{CDI},{ }^{108}$ the overall success rate of FMT was reported as $85 \%$ for recurrent $\mathrm{CDI}$ and $55 \%$ for refractory cases. Although the evidence for such is limited, FMT for CDI seems safe. In 2 RCTs, ${ }^{106,109}$ only mild adverse events such as diarrhea, cramping, belching, nausea, abdominal pain, and bloating were reported. ${ }^{106}$ In addition, the restored gut microbiota became similar to those of the donors, and persisted in a durable fashion after FMT. ${ }^{106,110,111}$ Thus, FMT is being applied to several diseases related with dysbiosis other than CDI. ${ }^{112}$ Although not nearly as effective as in CDI, FMT has also shown promising results in IBD. ${ }^{113-115} \mathrm{~A}$ far more complex microbial basis of the pathogenesis in IBD than in CDI, however, appears to need the best methodology, including ideal donor and recipient, mode of delivery, dose, and timing. ${ }^{116}$

The increasingly appreciated role of gut microbiota in the pathogenesis of FBDs and the imperfect current treatment can promote the use of FMT in selected patients with FBDs. ${ }^{117-119}$ To date, few case series have reported outcomes of FMT for constipation and IBS, ${ }^{120,121}$ but the results are promising. In a recent small study of FMT for refractory IBS (total of 13 patients: 9 IBS-D, 3 IBS$\mathrm{C}$, and 1 mixed IBS), a fecal suspension was infused into the distal duodenum or proximal jejunum via upper endoscopy. During a mean 11-month follow-up, resolution or alleviation of the symptoms was observed in $70 \%$ of the patients. In addition, the overall wellbeing was improved in $46 \%$ of the patients. The outcomes were not influenced by the donors, and only transient increased flatus was reported as an adverse event. These observations indicate that reconstituting the normal gut microbiota via FMT is a promising novel approach for FBD treatment, but further large studies, including well-designed RCTs, are mandatory to confirm that FMT is effective for FBD treatment. Although FMT appears to be well tolerated, a more specific modulation of the gut microbiota should be pursued in the future. Thus, the change in the gut microbiota in patients with FBDs after FMT also has to be investigated to characterize the specific microbial role of FMT for FBD treatment.

\section{Summary and Conclusion}

Dietary factors have strong effects on the gut microbiota. The composition of the gut microbiota, as affected by various diets, is related to the development of FBDs. At present, whether FBDs can be effectively treated or prevented by altering the composition of the gut microbiota through dietary modulation is still unknown. More research involving animal models, as well as robust epidemiologic and well-controlled interventional studies involving human beings are required.

Some probiotic strains that have been proven to be effective through RCTs can be a safe and promising treatment modality in FBD patients with IBS, and seems to be more effective in IBS-D than in IBS-C. Differentiation of the efficacy-proven strains and careful selection of the target patients based on both the symptoms and the properties of the available strains are important prerequisites of the clinical application of probiotics. A combination of specific probiotics and prebiotics (ie, synbiotics) may be an effective treatment option for IBS-C or FC in the future. Fermented milk products containing high concentrations of FODMAPs can aggravate the symptoms of IBS.

There is a consensus that rifaximin may be a treatment of choice for some IBS patients, but further studies are still warranted to identify the IBS patients who can best benefit from rifaximin use.

Reconstituting the normal gut microbiota via FMT is a promising novel approach for FBD treatment. Further large studies, however, including well-designed RCTs, are mandatory to confirm that FMT is effective for FBD treatment.

\section{Financial support: None.}

\section{Conflicts of interest: None.}

Author contributions: Hyo Jeong Lee, Jong Kyoung Choi, Yang Won Min, and Kyoung Sup Hong drafted the manuscript; Chang Hwan Choi, Eun Hee Kang, Han Seung Ryu, Kyung Sik Park, and Kyoung Sup Hong performed critical revision of the manuscript; and Yang Won Min and Kyoung Sup Hong coordinated the study and edited the manuscript. 


\section{References}

1. Longstreth GF, Thompson WG, Chey WD, Houghton LA, Mearin F, Spiller RC. Functional bowel disorders. Gastroenterology 2006;130:1480-1491.

2. Koloski NA, Talley NJ, Boyce PM. Epidemiology and health care seeking in the functional GI disorders: a population-based study. Am J Gastroenterol 2002;97:2290-2299.

3. Lovell RM, Ford AC. Global prevalence of and risk factors for irritable bowel syndrome: a meta-analysis. Clin Gastroenterol Hepatol 2012;10:712-721.e4.

4. Ohman L, Simrén M. Pathogenesis of IBS: role of inflammation, immunity and neuroimmune interactions. Nat Rev Gastroenterol Hepatol 2010;7:163-173.

5. Camilleri M, Lasch K, Zhou W. Irritable bowel syndrome: methods, mechanisms, and pathophysiology. The confluence of increased permeability, inflammation, and pain in irritable bowel syndrome. Am J Physiol Gastrointest Liver Physiol 2012;303:G775-G785.

6. Simrén M, Barbara G, Flint $H J$, et al. Intestinal microbiota in functional bowel disorders: a Rome foundation report. Gut 2013;62:159-176.

7. Spiller R, Lam C. An update on post-infectious irritable bowel syndrome: role of genetics, immune activation, serotonin and altered microbiome. J Neurogastroenterol Motil 2012;18:258-268.

8. Quigley EM. Gut bacteria in health and disease. Gastroenterol Hepatol (N Y) 2013;9:560-569.

9. Ford AC, Spiegel BM, Talley NJ, Moayyedi P. Small intestinal bacterial overgrowth in irritable bowel syndrome: systematic review and metaanalysis. Clin Gastroenterol Hepatol 2009;7:1279-1286.

10. Kassinen A, Krogius-Kurikka L, Mäkivuokko H, et al. The fecal microbiota of irritable bowel syndrome patients differs significantly from that of healthy subjects. Gastroenterology 2007;133:24-33.

11. Rajilić-Stojanović M, Biagi E, Heilig HG, et al. Global and deep molecular analysis of microbiota signatures in fecal samples from patients with irritable bowel syndrome. Gastroenterology 2011;141:1792-1801.

12. Jeffery IB, O’Toole PW, Öhman L, et al. An irritable bowel syndrome subtype defined by species-specific alterations in faecal microbiota. Gut 2012;61:997-1006

13. Parkes GC, Brostoff J, Whelan K, Sanderson JD. Gastrointestinal microbiota in irritable bowel syndrome: their role in its pathogenesis and treatment. Am J Gastroenterol 2008;103:1557-1567.

14. Claesson MJ, Jeffery IB, Conde S, et al. Gut microbiota composition correlates with diet and health in the elderly. Nature 2012;488:178-184.

15. Graf D, Di Cagno R, Fåk F, et al. Contribution of diet to the composition of the human gut microbiota. Microb Ecol Health Dis 2015;26:26164.

16. Conlon MA, Bird AR. The impact of diet and lifestyle on gut microbiota and human health. Nutrients 2015;7:17-44.

17. Flint HJ, Scott KP, Louis P, Duncan SH. The role of the gut microbiota in nutrition and health. Nat Rev Gastroenterol Hepatol 2012;9:577589.
18. Marchesi JR, Adams DH, Fava F, et al. The gut microbiota and host health: a new clinical frontier. Gut 2016;65:330-339.

19. Simrén M, Månsson A, Langkilde AM, et al. Food-related gastrointestinal symptoms in the irritable bowel syndrome. Digestion 2001;63:108115.

20. Böhn L, Störsrud S, Törnblom H, Bengtsson U, Simrén M. Selfreported food-related gastrointestinal symptoms in IBS are common and associated with more severe symptoms and reduced quality of life. Am J Gastroenterol 2013;108:634-641.

21. Hayes P, Corish C, O'Mahony E, Quigley EM. A dietary survey of patients with irritable bowel syndrome. J Hum Nutr Diet 2014;27(suppl 2):36-47.

22. Monsbakken KW, Vandvik PO, Farup PG. Perceived food intolerance in subjects with irritable bowel syndrome--etiology, prevalence and consequences. Eur J Clin Nutr 2006;60:667-672.

23. Hayes PA, Fraher MH, Quigley EM. Irritable bowel syndrome: the role of food in pathogenesis and management. Gastroenterol Hepatol (N Y) 2014;10:164-174.

24. McKenzie YA, Alder A, Anderson W, et al. British Dietetic Association evidence-based guidelines for the dietary management of irritable bowel syndrome in adults. J Hum Nutr Diet 2012;25:260-274.

25. Gibson PR, Varney J, Malakar S, Muir JG. Food components and irritable bowel syndrome. Gastroenterology 2015;148:1158-1174.e4.

26. Halmos EP, Power VA, Shepherd SJ, Gibson PR, Muir JG. A diet low in FODMAPs reduces symptoms of irritable bowel syndrome. Gastroenterology 2014;146:67-75.e5.

27. Böhn L, Störsrud S, Liljebo T, et al. Diet low in FODMAPs reduces symptoms of irritable bowel syndrome as well as traditional dietary advice: a randomized controlled trial. Gastroenterology 2015;149:13991407.e2.

28. Staudacher HM, Irving PM, Lomer MC, Whelan K. Mechanisms and efficacy of dietary FODMAP restriction in IBS. Nat Rev Gastroenterol Hepatol 2014;11:256-266.

29. Ong DK, Mitchell SB, Barrett JS, et al. Manipulation of dietary short chain carbohydrates alters the pattern of gas production and genesis of symptoms in irritable bowel syndrome. J Gastroenterol Hepatol 2010;25:1366-1373.

30. Zhu Y, Zheng X, Cong Y, et al. Bloating and distention in irritable bowel syndrome: the role of gas production and visceral sensation after lactose ingestion in a population with lactase deficiency. Am J Gastroenterol 2013;108:1516-1525.

31. Manichanh C, Eck A, Varela E, et al. Anal gas evacuation and colonic microbiota in patients with flatulence: effect of diet. Gut 2014;63:401408.

32. Pimentel M, Lin HC, Enayati P, et al. Methane, a gas produced by enteric bacteria, slows intestinal transit and augments small intestinal contractile activity. Am J Physiol Gastrointest Liver Physiol 2006;290:G1089-1095.

33. Pozuelo M, Panda S, Santiago A, et al. Reduction of butyrate- and methane-producing microorganisms in patients with irritable bowel syndrome. Sci Rep 2015;5:12693.

34. Triantafyllou K, Chang C, Pimentel M. Methanogens, methane and 
gastrointestinal motility. J Neurogastroenterol Motil 2014;20:31-40.

35. Kim G, Deepinder F, Morales W, et al. Methanobrevibacter smithii is the predominant methanogen in patients with constipation-predominant IBS and methane on breath. Dig Dis Sci 2012;57:3213-3218.

36. Tana C, Umesaki Y, Imaoka A, Handa T, Kanazawa M, Fukudo S. Altered profiles of intestinal microbiota and organic acids may be the origin of symptoms in irritable bowel syndrome. Neurogastroenterol Motil 2010;22:512-519, e114-115.

37. Staudacher HM, Lomer MC, Anderson JL, et al. Fermentable carbohydrate restriction reduces luminal bifidobacteria and gastrointestinal symptoms in patients with irritable bowel syndrome. J Nutr 2012;142:1510-1518.

38. Halmos EP, Christophersen CT, Bird AR, Shepherd SJ, Gibson PR, Muir JG. Diets that differ in their FODMAP content alter the colonic luminal microenvironment. Gut 2015;64:93-100.

39. Feinle-Bisset C, Azpiroz F. Dietary lipids and functional gastrointestinal disorders. Am J Gastroenterol 2013;108:737-747.

40. Faresjö A, Johansson S, Faresjö T, Roos S, Hallert C. Sex differences in dietary coping with gastrointestinal symptoms. Eur J Gastroenterol Hepatol 2010;22:327-333.

41. Serra J, Salvioli B, Azpiroz F, Malagelada JR. Lipid-induced intestinal gas retention in irritable bowel syndrome. Gastroenterology 2002;123:700-706.

42. Simrén M, Abrahamsson H, Björnsson ES. An exaggerated sensory component of the gastrocolonic response in patients with irritable bowel syndrome. Gut 2001;48:20-27.

43. Barbara G. Mucosal barrier defects in irritable bowel syndrome. Who left the door open? Am J Gastroenterol 2006;101:1295-1298.

44. Ghosh S, Molcan E, DeCoffe D, Dai C, Gibson DL. Diets rich in n-6 PUFA induce intestinal microbial dysbiosis in aged mice. Br J Nutr 2013;110:515-523.

45. Hildebrandt MA, Hoffmann C, Sherrill-Mix SA, et al. High-fat diet determines the composition of the murine gut microbiome independently of obesity. Gastroenterology 2009;137:1716-1724.e1-2.

46. Ding S, Chi MM, Scull BP, et al. High-fat diet: bacteria interactions promote intestinal inflammation which precedes and correlates with obesity and insulin resistance in mouse. PLoS One 2010;5:e12191.

47. Martinez-Medina M, Denizot J, Dreux N, et al. Western diet induces dysbiosis with increased $\mathrm{E}$ coli in CEABAC10 mice, alters host barrier function favouring AIEC colonisation. Gut 2014;63:116-124.

48. Duboc H, Rainteau D, Rajca S, et al. Increase in fecal primary bile acids and dysbiosis in patients with diarrhea-predominant irritable bowel syndrome. Neurogastroenterol Motil 2012;24:513-520, e246-247.

49. Appleby RN, Walters JR. The role of bile acids in functional GI disorders. Neurogastroenterol Motil 2014;26:1057-1069.

50. Devkota S, Wang Y, Musch MW, et al. Dietary-fat-induced taurocholic acid promotes pathobiont expansion and colitis in $I 110^{-/}$mice. Nature 2012;487:104-108.

51. Shahbazkhani B, Sadeghi A, Malekzadeh R, et al. Non-celiac gluten sensitivity has narrowed the spectrum of irritable bowel syndrome: a double-blind randomized placebo-controlled trial. Nutrients $2015 ; 7: 4542-4554$.
52. Biesiekierski JR, Newnham ED, Irving PM, et al. Gluten causes gastrointestinal symptoms in subjects without celiac disease: a double-blind randomized placebo-controlled trial. Am J Gastroenterol 2011;106:508514.

53. Vazquez-Roque MI, Camilleri M, Smyrk T, et al. A controlled trial of gluten-free diet in patients with irritable bowel syndrome-diarrhea: effects on bowel frequency and intestinal function. Gastroenterology 2013;144:903-911.e3.

54. Lee D, Albenberg L, Compher C, et al. Diet in the pathogenesis and treatment of inflammatory bowel diseases. Gastroenterology 2015;148:1087-1106.

55. Klingberg TD, Budde BB. The survival and persistence in the human gastrointestinal tract of five potential probiotic lactobacilli consumed as freeze-dried cultures or as probiotic sausage. Int J Food Microbiol 2006;109:157-159.

56. Ford AC, Quigley EM, Lacy BE, et al. Efficacy of prebiotics, probiotics, and synbiotics in irritable bowel syndrome and chronic idiopathic constipation: systematic review and meta-analysis. Am J Gastroenterol 2014;109:1547-1561.

57. Hungin AP, Mulligan C, Pot B, et al. Systematic review: probiotics in the management of lower gastrointestinal symptoms in clinical practice -- an evidence-based international guide. Aliment Pharmacol Ther 2013;38:864-886.

58. Ducrotté P, Sawant P, Jayanthi V. Clinical trial: Lactobacillus plantarum 299v (DSM 9843) improves symptoms of irritable bowel syndrome. World J Gastroenterol 2012;18:4012-4018.

59. Nobaek S, Johansson ML, Molin G, Ahrné S, Jeppsson B. Alteration of intestinal microflora is associated with reduction in abdominal bloating and pain in patients with irritable bowel syndrome. Am J Gastroenterol 2000;95:1231-1238.

60. Whorwell PJ, Altringer L, Morel J, et al. Efficacy of an encapsulated probiotic Bifidobacterium infantis 35624 in women with irritable bowel syndrome. Am J Gastroenterol 2006;101:1581-1590.

61. Macsharry J, O’Mahony L, Fanning A, et al. Mucosal cytokine imbalance in irritable bowel syndrome. Scand J Gastroenterol 2008;43:14671476.

62. Kim HJ, Camilleri M, McKinzie S, et al. A randomized controlled trial of a probiotic, VSL\#3, on gut transit and symptoms in diarrhoeapredominant irritable bowel syndrome. Aliment Pharmacol Ther 2003;17:895-904.

63. Kim HJ, Vazquez Roque MI, Camilleri M, et al. A randomized controlled trial of a probiotic combination VSL\# 3 and placebo in irritable bowel syndrome with bloating. Neurogastroenterol Motil 2005;17:687696.

64. Michail S, Kenche H. Gut microbiota is not modified by randomized, double-blind, placebo-controlled trial of VSL\#3 in diarrhea-predominant irritable bowel syndrome. Probiotics Antimicrob Proteins 2011;3:17.

65. Begtrup LM, de Muckadell OB, Kjeldsen J, Christensen RD, Jarbøl DE. Long-term treatment with probiotics in primary care patients with irritable bowel syndrome--a randomised, double-blind, placebo controlled trial. Scand J Gastroenterol 2013;48:1127-1135. 
66. Simrén M, Ohman L, Olsson J, et al. Clinical trial: the effects of a fermented milk containing three probiotic bacteria in patients with irritable bowel syndrome - a randomized, double-blind, controlled study. Aliment Pharmacol Ther 2010;31:218-227.

67. Sondergaard B, Olsson J, Ohlson K, Svensson U, Bytzer P, Ekesbo R. Effects of probiotic fermented milk on symptoms and intestinal flora in patients with irritable bowel syndrome: a randomized, placebo-controlled trial. Scand J Gastroenterol 2011;46:663-672.

68. Agrawal A, Houghton LA, Morris J, et al. Clinical trial: the effects of a fermented milk product containing Bifidobacterium lactis DN-173 010 on abdominal distension and gastrointestinal transit in irritable bowel syndrome with constipation. Aliment Pharmacol Ther 2009;29:104114.

69. Guyonnet D, Chassany O, Ducrotte P, et al. Effect of a fermented milk containing Bifidobacterium animalis DN-173 010 on the health-related quality of life and symptoms in irritable bowel syndrome in adults in primary care: a multicentre, randomized, double-blind, controlled trial. Aliment Pharmacol Ther 2007;26:475-486.

70. Ligaarden SC, Axelsson L, Naterstad K, Lydersen S, Farup PG. A candidate probiotic with unfavourable effects in subjects with irritable bowel syndrome: a randomised controlled trial. BMC Gastroenterol 2010;10:16.

71. Niv E, Naftali T, Hallak R, Vaisman N. The efficacy of Lactobacillus reuteri ATCC 55730 in the treatment of patients with irritable bowel syndrome--a double blind, placebo-controlled, randomized study. Clin Nutr 2005;24:925-931.

72. Kajander K, Hatakka K, Poussa T, Färkkilä M, Korpela R. A probiotic mixture alleviates symptoms in irritable bowel syndrome patients: a controlled 6-month intervention. Aliment Pharmacol Ther 2005;22:387394.

73. Marteau P, Cuillerier E, Meance S, et al. Bifidobacterium animalis strain DN-173 010 shortens the colonic transit time in healthy women: a double-blind, randomized, controlled study. Aliment Pharmacol Ther 2002;16:587-593.

74. Krammer HJ, von Seggern H, Schaumburg J, Neumer F. Effect of Lactobacillus casei Shirota on colonic transit time in patients with chronic constipation. coloproctology 2011;33:109-113.

75. Waller PA, Gopal PK, Leyer GJ, et al. Dose-response effect of Bifidobacterium lactis HN019 on whole gut transit time and functional gastrointestinal symptoms in adults. Scand J Gastroenterol 2011;46:10571064.

76. Favretto DC, Pontin B, Moreira TR. Effect of the consumption of a cheese enriched with probiotic organisms (Bifidobacterium lactis Bi-07) in improving symptoms of constipation. Arq Gastroenterol 2013;50:196201.

77. Mazlyn MM, Nagarajah LH, Fatimah A, Norimah AK, Goh KL. Effects of a probiotic fermented milk on functional constipation: a randomized, double-blind, placebo-controlled study. J Gastroenterol Hepatol 2013;28:1141-1147.

78. Ojetti V, Ianiro G, Tortora A, et al. The effect of Lactobacillus reuteri supplementation in adults with chronic functional constipation: a randomized, double-blind, placebo-controlled trial. J Gastrointestin Liver
Dis 2014;23:387-391.

79. Riezzo G, Orlando A, D'Attoma B, et al. Randomised clinical trial: efficacy of Lactobacillus paracasei-enriched artichokes in the treatment of patients with functional constipation--a double-blind, controlled, crossover study. Aliment Pharmacol Ther 2012;35:441-450.

80. Boyle RJ, Robins-Browne RM, Tang ML. Probiotic use in clinical practice: what are the risks? Am J Clin Nutr 2006;83:1256-1264.

81. Quigley EM. Prebiotics and probiotics: their role in the management of gastrointestinal disorders in adults. Nutr Clin Pract 2012;27:195-200.

82. Kunz AN, Noel JM, Fairchok MP. Two cases of Lactobacillus bacteremia during probiotic treatment of short gut syndrome. J Pediatr Gastroenterol Nutr 2004;38:457-458.

83. Besselink MG, van Santvoort HC, Buskens E, et al. Probiotic prophylaxis in predicted severe acute pancreatitis: a randomised, double-blind, placebo-controlled trial. Lancet 2008;371:651-659.

84. Halmos EP, Power VA, Shepherd SJ, Gibson PR, Muir JG. A diet low in FODMAPs reduces symptoms of irritable bowel syndrome. Gastroenterology 2014;146:67-75.e5.

85. Pimentel M. Review article: potential mechanisms of action of rifaximin in the management of irritable bowel syndrome with diarrhoea. Aliment Pharmacol Ther 2016;43(suppl 1):37-49.

86. Yang J, Lee HR, Low K, Chatterjee S, Pimentel M. Rifaximin versus other antibiotics in the primary treatment and retreatment of bacterial overgrowth in IBS. Dig Dis Sci 2008;53:169-174.

87. Basseri RJ, Weitsman S, Barlow GM, Pimentel M. Antibiotics for the treatment of irritable bowel syndrome. Gastroenterol Hepatol (N Y) 2011;7:455-493.

88. Marchese A, Salerno A, Pesce A, Debbia EA, Schito GC. In vitro activity of rifaximin, metronidazole and vancomycin against Clostridium difficile and the rate of selection of spontaneously resistant mutants against representative anaerobic and aerobic bacteria, including ammoniaproducing species. Chemotherapy 2000;46:253-266.

89. Phongsamran PV, Kim JW, Cupo Abbott J, Rosenblatt A. Pharmacotherapy for hepatic encephalopathy. Drugs 2010;70:1131-1148.

90. Scarpignato C, Pelosini I. Rifaximin, a poorly absorbed antibiotic: pharmacology and clinical potential. Chemotherapy 2005;51(suppl 1):36-66.

91. DuPont HL. Review article: the antimicrobial effects of rifaximin on the gut microbiota. Aliment Pharmacol Ther 2016;43(suppl 1):3-10.

92. Sharara AI, Aoun E, Abdul-Baki H, Mounzer R, Sidani S, Elhajj I. A randomized double-blind placebo-controlled trial of rifaximin in patients with abdominal bloating and flatulence. Am J Gastroenterol 2006;101:326-333.

93. Pimentel M, Park S, Mirocha J, Kane SV, Kong Y. The effect of a nonabsorbed oral antibiotic (rifaximin) on the symptoms of the irritable bowel syndrome: a randomized trial. Ann Intern Med 2006;145:557563.

94. Menees SB, Maneerattannaporn M, Kim HM, Chey WD. The efficacy and safety of rifaximin for the irritable bowel syndrome: a systematic review and meta-analysis. Am J Gastroenterol 2012;107:28-35.

95. Gupta A, Chey WD. Breath testing for small intestinal bacterial overgrowth: a means to enrich rifaximin responders in IBS patients? Am J Gastroenterol 2016;111:305-306. 
96. Peralta S, Cottone C, Doveri T, Almasio PL, Craxi A. Small intestine bacterial overgrowth and irritable bowel syndrome-related symptoms: experience with rifaximin. World J Gastroenterol 2009;15:2628-2631.

97. Pimentel M, Chow EJ, Lin HC. Normalization of lactulose breath testing correlates with symptom improvement in irritable bowel syndrome: a double-blind, randomized, placebo-controlled study. Am J Gastroenterol 2003;98:412-419.

98. Ghoshal UC, Srivastava D. Irritable bowel syndrome and small intestinal bacterial overgrowth: meaningful association or unnecessary hype. World J Gastroenterol 2014;20:2482-2491.

99. Kasir R, Zakko S, Zakko P, et al. Predicting a response to antibiotics in patients with the irritable bowel syndrome. Dig Dis Sci 2016;61:846851.

100. Ghoshal UC, Srivastava D, Misra A, Ghoshal U. A proof-of-concept study showing antibiotics to be more effective in irritable bowel syndrome with than without small-intestinal bacterial overgrowth: a randomized, double-blind, placebo-controlled trial. Eur J Gastroenterol Hepatol 2016;28:281-289.

101. Mullen KD, Sanyal AJ, Bass NM, et al. Rifaximin is safe and well tolerated for long-term maintenance of remission from overt hepatic encephalopathy. Clin Gastroenterol Hepatol 2014;12:1390-1397.e2.

102. Carman RJ, Boone JH, Grover H, Wickham KN, Chen L. In vivo selection of rifamycin-resistant Clostridium difficile during rifaximin therapy. Antimicrob Agents Chemother 2012;56:6019-6020.

103. Kothary V, Scherl EJ, Bosworth B, et al. Rifaximin resistance in Escherichia coli associated with inflammatory bowel disease correlates with prior rifaximin use, mutations in $r p o B$, and activity of Phe-Arg- $\beta$ naphthylamide-inhibitable efflux pumps. Antimicrob Agents Chemother 2013;57:811-817.

104. Valentin T, Leitner E, Rohn A, et al. Rifaximin intake leads to emergence of rifampin-resistant staphylococci. J Infect 2011;62:34-38.

105. Cammarota G, Ianiro G, Gasbarrini A. Fecal microbiota transplantation for the treatment of Clostridium difficile infection: a systematic review. J Clin Gastroenterol 2014;48:693-702.

106. van Nood E, Vrieze A, Nieuwdorp M, et al. Duodenal infusion of donor feces for recurrent Clostridium difficile. N Engl J Med 2013;368:407-415.

107. Kassam Z, Lee CH, Yuan Y, Hunt RH. Fecal microbiota transplantation for Clostridium difficile infection: systematic review and metaanalysis. Am J Gastroenterol 2013;108:500-508.

108. Drekonja D, Reich J, Gezahegn S, et al. Fecal microbiota transplantation for Clostridium difficile infection: a systematic review. Ann Intern Med 2015;162:630-638

109. Youngster I, Sauk J, Pindar C, et al. Fecal microbiota transplant for relapsing Clostridium difficile infection using a frozen inoculum from unrelated donors: a randomized, open-label, controlled pilot study. Clin Infect Dis 2014;58:1515-1522.

110. Khoruts A, Dicksved J, Jansson JK, Sadowsky MJ. Changes in the composition of the human fecal microbiome after bacteriotherapy for recurrent Clostridium difficile-associated diarrhea. J Clin Gastroenterol 2010;44:354-360.

111. Hamilton MJ, Weingarden AR, Unno T, Khoruts A, Sadowsky MJ. High-throughput DNA sequence analysis reveals stable engraftment of gut microbiota following transplantation of previously frozen fecal bacteria. Gut Microbes 2013;4:125-135.

112. Kelly CR, Kahn S, Kashyap P, et al. Update on fecal microbiota transplantation 2015: indications, methodologies, mechanisms, and outlook. Gastroenterology 2015;149:223-237.

113. Colman RJ, Rubin DT. Fecal microbiota transplantation as therapy for inflammatory bowel disease: a systematic review and meta-analysis. J Crohns Colitis 2014;8:1569-1581.

114. Moayyedi P, Surette MG, Kim PT, et al. Fecal microbiota transplantation induces remission in patients with active ulcerative colitis in a randomized controlled trial. Gastroenterology 2015;149:102-109.e6.

115. Rossen NG, Fuentes S, van der Spek MJ, et al. Findings from a randomized controlled trial of fecal transplantation for patients with ulcerative colitis. Gastroenterology 2015;149:110-118.e4.

116. Grinspan AM, Kelly CR. Fecal microbiota transplantation for ulcerative colitis: not just yet. Gastroenterology 2015;149:15-18.

117. Saulnier DM, Ringel Y, Heyman MB, et al. The intestinal microbiome, probiotics and prebiotics in neurogastroenterology. Gut Microbes 2013;4:17-27.

118. Camilleri M. Peripheral mechanisms in irritable bowel syndrome. N Engl J Med 2012;367:1626-1635.

119. Bennet SM, Ohman L, Simren M. Gut microbiota as potential orchestrators of irritable bowel syndrome. Gut Liver 2015;9:318-331.

120. Pinn DM, Aroniadis OC, Brandt LJ. Is fecal microbiota transplantation (FMT) an effective treatment for patients with functional gastrointestinal disorders (FGID)? Neurogastroenterol Motil 2015;27:19-29.

121. Pinn DM, Aroniadis OC, Brandt LJ. Is fecal microbiota transplantation the answer for irritable bowel syndrome? A single-center experience. Am J Gastroenterol 2014;109:1831-1832. 